doi: $10.15407 /$ ukrbotj73.03.273

\author{
A.Ye. KHODOSOVTSEV ${ }^{1}$, V.V. DARMOSTUK ${ }^{1}$, A.B. GROMAKOVA ${ }^{2}$ \\ ${ }^{1}$ Kherson State University \\ 27, Universytetska Str., Kherson, 73000, Ukraine \\ khodosovtsev@i.ua \\ ${ }^{2}$ V.N. Karazin Kharkiv National University \\ 4, Svobody Sq., Kharkiv, 61022, Ukraine \\ gromakova@mail.ru
}

\title{
NEW FOR UKRAINE LICHEN-FORMING AND LICHENICOLOUS FUNGI FROM GORGANY NATURE RESERVE
}

Khodosovtsev A.Ye., Darmostuk V.V., Gromakova A.B. New for Ukraine lichen-forming and lichenicolous fungi from Gorgany Nature Reserve. Ukr. Bot. J., 2016, 73(3): 273-276.

Abstract. Six new for Ukraine species of lichen-forming (Caloplaca borreri, Micarea viridiatra, Micarea viridileprosa, Porina guentheri, Schismatomma cretaceum, Xylographa vitiligo) and three species of lichenicolous fungi (Didymocyrtis pseudeverniae, Milospilum lacoizquetae, Psammina stipitata) from Gorgany Nature Reserve are reported. The materials were collected within the area of primeval spruce forests on bark of Acer pseudoplatanus, Abies alba, Fagus sylvatica, Pinus cembra, as well as on sandstone outcrops, wood and different lichens. The descriptions, localities, ecological and distribution data for these species are provided.

Key words: Carpathians, primeval coniferous forests, bark, sandstone

\section{Introduction}

Gorgany Nature Reserve located in south-west part of Ivano-Frankivsk Region covers 5344 ha in the Dovbushanski Gorgany Mountains. The forest with Picea abies (L.) H. Karst is a predominant type of vegetation which occupies $84 \%$ of its area (Klimuk et al., 2006). These are the most valuable areas of primeval coniferous forests in Ukraine. Special features of these Picea forests are admixtures of Acer pseudoplatanus L., Abies alba Mill., Fagus sylvatica L., and Pinus cembra L. Moreover, the sandstone outcrops cover some slopes in different parts of the reserve. Several mountain belts, variety of forests types and substrates provide the preconditions for a high diversity of lichen-forming and lichenicolous fungi. Unfortunately, sparse information about lichen-forming and lichenicolous fungi included in «The Chronicle of Nature» of the Reserve has not been published before. The first information about nine new for Ukraine lichenized and lichenicolous fungi collected in Gorgany Nature Reserve is provided in this paper.

\section{Materials and methods}

The materials were collected during special expedition in Gorgany Nature Reserve in May 2015 by the authors of the paper. All specimens were examined using standard microscope techniques and deposited

(C) A.Ye. KHODOSOVTSEV, V.V. DARMOSTUK,

A.B. GROMAKOVA, 2016 in the Lichenological Herbarium of Kherson State University $(K H E R)$. A list of species is given below. The lichenicolous fungi are marked by asterisk $\ll^{*} »$.

\section{Results}

Caloplaca borreri J.R. Laundon

Thallus of small leprose portions without areoles, yellow-brownish, soredia 40-50 $\mu \mathrm{m}$ diam., $\mathrm{K}+$ purple. Apothecia unknown.

SUBSTRATE. On bark at the base of an old Acer psedoplatanus tree.

SPECIMEN EXAMINED. Ukraine. IvanoFrankivsk Region, Nadvirna District, Gorgany Nature Reserve, sq. 10 , plot $1,48^{\circ} 47507^{\prime} \mathrm{N} 024^{\circ} 30680^{\prime} \mathrm{E}$, alt. 995 m, 5 May 2015, A. Khodosovtsev, V. Darmostuk, A. Gromakova (KHER 9084).

Previously known only from four locations in England (Laundon, 2005). It is for the first time reported for Eastern Europe.

*Didymocyrtis pseudeverniae (Etayo \& Diederich) Ertz \& Diederich

Conidiomata immersed in thallus of Pseudevernia furfuracea (L.) Zopf, 130-170 $\mu \mathrm{m}$ diam., conidia hyaline, ellipsoid, sometimes irregularly shaped, (14-)16$22(-26) \times 6-9 \mu \mathrm{m}$.

SUBSTRATE. On Pseudevernia furfuracea, on Picea abies.

SPECIMEN EXAMINED. Ukraine. IvanoFrankivsk Region, Nadvirna District, Gorgany Nature 
Reserve, near cordon, $48^{\circ} 48517^{\prime} \mathrm{N} 024^{\circ} 28504^{\prime} \mathrm{E}$, alt. 810 m, 5 May 2015, A. Khodosovtsev, V. Darmostuk, A. Gromakova (KHER 9085).

This lichenicolous fungus was hitherto found in Czech Republic (Brackel, 2009), Germany (Brackel, 2014), Great Britain (Hawksworth, 2003), Lithuania (Motiejūnaitè et al., 2011), and Spain (Etayo, Diederich, 1996).

\section{Micarea viridiatra Coppins}

Thallus crustose, often with granular areoles, 60$120 \mu \mathrm{m}$ diam, greenish. Apothecia $0.15-0.3 \mathrm{~mm}$ diam., convex, greenish, hymenium and hypothecium bluegreen, ascospores hyaline, $0-1$ septate, (10-) 12-17 $(-19) \times(3.5)-4-5 \mu \mathrm{m}$.

SUBSTRATE. On wood.

SPECIMEN EXAMINED. Ukraine. IvanoFrankivsk Region, Nadvirna District, Gorgany Nature Reserve, Dzhurdzhinets stream, 48 $48384^{\prime} \mathrm{N}$ $024^{\circ} 27692^{\prime} \mathrm{E}$, alt. 815 m, 2 May 2015, A. Khodosovtsev, V. Darmostuk, A. Gromakova (KHER 8884).

In Europe it is known from Great Britain and Norway (Smith et al., 2009).

Micarea viridileprosa Coppins \& Van den Boom

Thallus crustose, wide-spread, diffuse, bright green, composed of discrete, bright greenish goniocysts. Goniocysts $12-18 \mu \mathrm{m}$ diam., photobiont micareoid cells 4-7 $\mu \mathrm{m}$ diam. Apothecia in Ukrainian material absent. Thallus $\mathrm{C}+$ red, $\mathrm{K}-, \mathrm{KC}+$ red, $\mathrm{Pd}-$.

SUBSTRATE. On bark of Picea abies.

SPECIMEN EXAMINED. Ukraine. IvanoFrankivsk Region, Nadvirna District, Gorgany Nature Reserve, sq. 10 , plot $1,48^{\circ} 47507^{\prime} \mathrm{N} 024^{\circ} 30680^{\prime} \mathrm{E}$, alt. 995 m, 5 May 2015, A. Khodosovtsev, V. Darmostuk, A. Gromakova (KHER 8898).

It is rather common species in the following European countries: Belgium, Great Britain, France, Germany, Italy, the Netherlands, Portugal, Switzerland (Boom \& Coppins, 2001), Czech Republic (Malicek et al., 2014), Norway (Tønsberg, Johnsen, 2011), Poland (Czarnota, 2007), Russia (Urbanavichus, 2010), and Sweden (Thor, 2009). It is also recorded from Tasmania (Smith et al., 2009).

* Milospium lacoizquetae Etayo \& Diederich

Conidiomata sporodochia, (30-)50-100(-120) $\mu \mathrm{m}$ wide, flattened, brownish; conidia aseptate, sometimes irregularly folded and lobate (fine visible in $\mathrm{K}$ ), dark brown, $7-14(-16) \times 6-10(-12) \mu \mathrm{m}$.
SUBSTRATE. On squamules on epiphytic Cladonia sp., on Picea abies.

SPECIMEN EXAMINED. Ukraine. IvanoFrankivsk Region, Nadvirna District, Gorgany Nature Reserve, sq. 11 , near cordon 15 locality, $48^{\circ} 47446^{\prime} \mathrm{N}$ 02430808'E, alt. 995 m, 5 May 2015, A. Khodosovtsev, V. Darmostuk, A. Gromakova (KHER 8970).

This lichenicolous fungus is known from a few localities in Austria (Hafellner et al., 2004), Czech Republic (Kocourkova et al., 2005), France (Etayo, Diederich, 1996), Germany (Brackel, 2006), Poland (Schiefelbein et al., 2012), and Spain (Etayo, 2002).

Porina guentheri (Flot.) Zahlbr.

Thallus continuous, dull green-brown. Perithecia black, 0.3-0.7 mm diam., involucrellum purple-brown. Ascospores (6-)7(-9)-septate, (28-)32-45(49) $\times$ (4.5) $5-6-5 \mu \mathrm{m}$

SUBSTRATE. On shaded sandstone.

SPECIMEN EXAMINED. Ukraine. IvanoFrankivsk Region, Nadvirna District, Gorgany Nature Reserve, sq. 11, near beech trees numbers 265-266, $48^{\circ} 48703^{\prime} \mathrm{N} 024^{\circ} 28640^{\prime} \mathrm{E}$, alt. $880 \mathrm{~m}, 1$ May 2015, A. Khodosovtsev, V. Darmostuk, A. Gromakova (KHER 8637).

It is known locally from Europe, Asia, North America, South Africa and Australia (Tasmania) (Smith et al., 2009).

* Psammina stipitata D. Hawksw.

Colonies discrete, composed of hyaline to pale brown torulose hyphae mainly 3-4 $\mu \mathrm{m}$ wide. Conidiophores arising in small groups, straight, unbranched, smoothwalled, septate, $20-50 \times 4-5 \mu \mathrm{m}$. Conidiogenous cells monRegionic, integrated, terminal, cylindrical. Conidia acrogenous, multiseptate, palmate to cartwheel-like, brown in mass, comprising about 50 arms, overall 50 $80 \mu \mathrm{m}$ diam; individual arms straight or slightly curved, 3-7 euseptate, often attenuated at the apex, subhyaline to pale brown, smooth or irregularly sparsely rugose, $25-50 \times 3-3.5(-4.5) \mu \mathrm{m}$.

SUBSTRATE. On green algae and Lecanora sp. on wood.

SPECIMEN EXAMINED. Ukraine. IvanoFrankivsk Region, Nadvirna District, Gorgany Nature Reserve, sq. $11,48^{\circ} 46411^{\prime} \mathrm{N} 024^{\circ} 31678^{\prime} \mathrm{E}$, alt. 1400 m, 5 May 2015, A. Khodosovtsev, V. Darmostuk, A. Gromakova (KHER 9086).

It was hitherto known from France (Coste, 2012), England (Earland-Bennet, Hawksworth, 1999), 
the Netherlands (van den Boom, 2009) and Spain (Navarro-Rosinés et al., 1994).

Schismatomma cretaceum (Hue) J.R. Laundon

Thallus crustose, whitish, sorediate. Soralia delimited to irregular, convex, chalk-white. Apothecia and pycnidia unknown. Thallus $\mathrm{C}-, \mathrm{K}+$ yellowish, $\mathrm{KC}-, \mathrm{Pd}-$.

SUBSTRATE. On dry side of old Acer psedoplatanus.

SPECIMEN EXAMINED. Ukraine. IvanoFrankivsk Region, Nadvirna District, Gorgany Nature Reserve, sq. 10, plot $1,48^{\circ} 47507^{\prime} \mathrm{N} 024^{\circ} 30680^{\prime} \mathrm{E}$, alt. 995 m, 5 May 2015, A. Khodosovtsev, V. Darmostuk, A. Gromakova (KHER 9087).

This rare lichen-forming fungus was found so far in a few localities in Western Europe (Great Britain) (Smith et al., 2009).

Xylographa vitiligo (Ach.) J.R. Laundon

Thallus immersed, pale greyish, soralia present, discrete, $0.2-1 \times 0.18-0.42 \mathrm{~mm}$, flat, dark grey to cream; soredia $20-30 \mu \mathrm{m}$ diam, with brown pigment which is $\mathrm{K}-$. Soralia $\mathrm{C}-, \mathrm{K}+$ yellow, $\mathrm{Pd}+$ orange. Apothecia in Ukrainian material not found.

SUBSTRATE. On coniferous wood.

SPECIMEN EXAMINED. Ukraine. IvanoFrankivsk Region, Nadvirna District, Gorgany Nature Reserve, sq. 10, alt. 1085 m, 48²6865'N 02430973'E, 5 May 2015, A. Khodosovtsev, V. Darmostuk, A. Gromakova (KHER 9089).

It is known from Europe, Asia, Macaronesia, North America, Africa, and subantarctic islands (Smith et al., 2009).

\section{Acknowledgement}

We are thankful to M.B. Shpilchak (Ivano-Frankivsk, Ukraine) for help during expedition in Gorgany Nature Reserve and to V.P. Heluta (Kyiv, Ukraine) for his assistance in communication with the reserve administration; to S.Ya. Kondratyuk and S.L. Mosyakin (Kyiv, Ukraine) for valuable comments and linguistic corrections. The fieldwork study was supported by Toyota Center Kherson «Auto-Plaza» and Mr. V. Borzhkov (Kherson, Ukraine).

\section{REFERENCES}

Brackel W. von. Weitere Funde von flechtenbewohnenden Pilzen in Bayern - Beitrag zu einer Checkliste IV, Berichte der Bayerischen Botanischen Gesellschaft, 2009, 11: $5-55$.
Brackel W. von. Kommentierter Katalog der flechtenbewohnenden Pilze Bayerns, Biblioth. Lichenol., 2014, 109: $1-476$.

Brackel W. von., Kocourková J. Endococcus karlstadtensis sp. nov. und weitere Funde von flechtenbewohnenden Pilzen in Bayern - Beitrag zu einer Checkliste II, Berichte der Bayerischen Botanischen Gesellschaft, 2006, 76: 5-32.

Boom P.P.G. van den, Coppins B.J. Micarea viridileprosa sp. nov., an overlooked lichen species from Western Europe, Lichenologist, 2001, 33: 87-91.

Boom P.P.G. van den. Diversity of lichens and lichenicolous fungi in a primeval heathland and adjacent managed forest in southern Netherlands (Groote Heide and 't Leenderbos), Öster. Z. Pilzk., 2009, 18: 25-45.

Coste $\mathrm{C}$. Inventaire raisonné des lichens et des champignons lichénicoles de la forêt domaniale de Grésigne (Tarn, France), Bull. Soc. Hist. Nat. Toulouse., 2012, 148: 3-12.

Czarnota P. The lichen genus Micarea (Lecanorales, Ascomycotina) in Poland, Polish botanical studies, 2007, 23: $1-199$.

Earland-Bennet P.M., Hawksworth D.L. Observations on the genus Psammia, including the description of two new algicolous and lichenicolous species, Lichenologist, 1999, 31(6): 579-586.

Etayo J., Diederich P. Lichenicolous fungi from the Western Pyrenees, France and Spain II. More Deuteromycetes, Mycotaxon, 1996, 60: 415-428.

Etayo J. Catálogo de líquenes y hongosliquenícolasdel Parque Naturel de Bértiz (Navarra, España), Bull. Soc. Linn. Provence, 2002, 53: 155-170.

Hafellner J., Kocourková J., Obermayer W. Records of lichenicolous fungi from the northern Schladminger Tauern (Eastern Alps, Austria, Styria), Herzogia, 2004, 17: 59-66.

Hawksworth D. The lichenicolous fungi of Great Britain and Ireland: an overview and annotated checklist, Lichenologist, 2003, 35(3): 191-232.

Klimuk Yu.V., Miskevych U.D., Yakushenko D.M., Chorney I.I., Budzhak V.V., Nyporko S.O., Shpilchak M.B., Chernavsky M.V., Tokaryuk A.I., Oleksiv T.M., Tymchuk Ya.Ya., Solomakha V.A. Solomakha T.D., Mayor R.V. Nature reserve «Gorgany». Plant World. Nature reserve territories of Ukraine. Plant Word. Iss. 6., Kyiv: Phytosociocentre, 2006, 400 рр. [Клімук Ю.В., Міскевич У.Д., Якушенко Д.М., Буджак В.В., Нипорко С.О. Шпільчак М.Б., Чернавський М.В., Токарюк А.І., Олексів Т.М., Тимчук Я.Я., Соломаха В.А., Соломаха Т.Д., Майор Р.В. Природний заповідник «Горгани». Природно-заповідні території України. Рослинний світ. Випуск VI, К.: Фітосоціоцентр, 2006, 400 с.]

Kocourková J., Boom P.P.G. van den. Lichenicolous fungi from the Czech Republic II. Arthrorhaphis arctoparmeliae spec. nov. and some new records for the country, Herzogia, 2005, 18: 23-35.

Laundon J.R. The publication and typification of Sir James Edward Smith's lichens in English Botany, Botanical Journal of the Linnean Society, 2005, 147(4): 483-499. 
Malíček J., Palice Z., Vondràk J. New lichen records and rediscoveries from the Czech Republic and Slovakia, Herzogia, 2014, 27(2): 257-284.

Motiejūnaitė J., Brackel W. von, Stončius D., Preikša Ž. Contribution to the Lithuanian flora of lichens and allied fungi, III, Botanica Lithuanica, 2011, 17(1): 39-46.

Navarro-Rosinés P., Boqueras M., Llimona X. Primer cataleg dels fongs liquenicoles de Catalunya i zones proximes (NE de la Peninsula Iberica), Bulletino Sociedad Catalana Micologia, 1994, 16-17: 165-204.

Schiefelbein U., Czarnota P., Thüs H., Kukwa M. The lichen biota of the Drawieński National Park (NW Poland, Western Pomerania), Folia Cryptog. Estonica, 2012, 49: 59-71.

Smith C.W., Aptroot B.J., Coppins B.J., Flecher A., Gilbert O.L., James P.W., Wolseley P.A. The Lichens of Great Britain and Ireland. Nat. Hist. Mus. Publ., 2009, 1046 pp.

Thor G. Micarea viridileprosa och M. botryoides I Sverige, Lavbulletinen, 2009, 2: 60-64.

Tønsberg T., Johnson J. Micarea viridileprosa new to Norway, Graphis Scripta, 2011, 23: 30.

Urbanavichus G. A checklist of the lichen flora of Russia. St. Petersburg: Nauka, 2010, 194 pp. [Урбанавичус Г. Сnuсок видов лихенофлоры России. СПб.: Наука, 2010, 194 c.]

Recommended by

Submitted 03.11.2015

V.P. Hayova
О.Є. Ходосовцев ${ }^{1}$, В.В. Дармостук ${ }^{1}$, А.Б. Громакова ${ }^{2}$. Нові для України види лишайників та ліхенофільних грибів 3 природного заповідника «Горгани». - Укр. ботан. журн. - 2016. - 73(3): 273-276.

${ }^{1}$ Херсонський державний університет вул. Університетська, 27, м. Херсон, 73000, Україна

${ }^{2}$ Харьківський національний університет імені В.Н. Каразіна пл. Свободи, 4, м. Харків, 61022, Україна

В статті наведено опис, місцезнаходження, екологічні особливості та загальне поширення нових для України шести видів лишайників (Caloplaca borreri, Micarea viridiatra, Micarea viridileprosa, Porina guentheri, Schismatomma cretaceum, Xylographa vitiligo) та трьох видів ліхенофільних грибів (Didymocyrtis pseudeverniae, Milospium lacoizquetae, Psammina stipitata), знайдених на території природного заповідника «Горгани».

Ключові слова: Карпати, хвойні праліси, кора, пісковики

А.Е. Ходосовцев ${ }^{1}$, В.В. Дармостук ${ }^{1}$, А.Б. Громакова ${ }^{2}$. Новые для Украины виды лишайников и лихенофильных грибов из природного заповедника «Горганы». - Укр. ботан. журн. - 2016. - 73(3): 273-276.

${ }^{1}$ Херсонский государственный университет ул. Университетская, 27, г. Херсон, 73000, Украина ${ }^{2}$ Харьковский национальный университет имени В.Н. Каразина пл. Свободы, 4, г. Харьков, 61022, Украина

В статье приведены описание, местонахождения, экологические особенности и общее распространение новых для Украины шести видов лишайников (Caloplaca borreri, Micarea viridiatra, Micarea viridileprosa, Porina guentheri, Schismatomma cretaceum, Xylographa vitiligo) и трех видов лихенофильных грибов (Didymocyrtis pseudeverniae, Milospium lacoizquetae, Psammina stipitata), найденных на территории природного заповедника «Горганы».

Ключевые слова: Карпаты, хвойные пралеса, кора, песчаники 